\title{
AVALIAÇÃO DA QUALIDADE DE VIDA EM PACIENTES SUBMETIDOS À CIRURGIA DE ESTENOSE DE CANAL LOMBAR COM MAIS DE 60 ANOS
}

\author{
EVALUATION OF QUALITY OF LIFE IN PATIENTS WITH OVER 60 YEARS UNDERGOING \\ SURGERY FOR LUMBAR CANAL STENOSIS
}

\author{
EVALUACIÓN DE LA CALIDAD DE VIDA EN PACIENTES SOMETIDOS A LA CIRUGÍA DE \\ ESTENOSIS DE CANAL LUMBAR, CON MÁS DE 60 AÑOS DE EDAD
}

Caio Vargas Yoshino", Luciano Miller Reis Rodrigues ${ }^{2}$

\begin{abstract}
RESUMO
Objetivo. Avaliar as mudanças na qualidade de vida antes e após a cirurgia de artrodese lombar através dos questionários Owestry Disablity Index (ODI), Roland-Morris (RM) e Escala Analógica Visual (VAS). Métodos. Foram avaliadas as mudanças nos questionários (ODI, RM e VAS) entre o pré-operatório, 1 semana, 1 mês, 2 meses, 6 meses, 1 ano e 2 anos após o procedimento cirúrgico. Resultados. Foram estudados 38 pacientes com idade acima de 60 anos com acompanhamento de pelo menos dois anos. Houve uma melhora no ODI de 55,94\%, 49,18 no RM e 39,48 no VAS. Conclusão. Os resultados mostram uma melhora nos índices analisados, apresentando índices semelhantes a estudos anteriores, apesar da discrepância entre os diversos estudos e à falta de dados referentes à nossa população.
\end{abstract}

Descritores: Artrodese; Vértebras lombares; Fusão vertebral; Qualidade de vida.

\begin{abstract}
Objective. Evaluate changes in quality of life before and after lumbar arthrodesis surgery by means of questionnaires Owestry Disablity Index (ODI), Roland-Morris (RM) and Visual Analogue Scale (VAS). Methods. We evaluated the changes in the questionnaires (ODI, RM and VAS) in the preoperative period, 1 week, 1 month, 2 months, 6 months, 1 year and 2 years after surgery. Results. We studied 38 patients aged over 60 with follow up of at least two years. An improvement of $55.94 \%$ in ODI, of 49.18\% in RM and 39.48\% in VAS was observed. Conclusion. The results show an improvement in the indices studied, with rates similar to previous studies, despite the discrepancy among the various studies and the lack of data on our population.
\end{abstract}

Keywords: Arthrodesis; Lumbar vertebrae; Spinal fusion; Quality of Life

\section{RESUMEN}

Objetivo. Evaluar los cambios en la calidad de vida, antes y después de la cirugía de artrodesis lumbar, mediante los cuestionarios Owestry Disablity Index (ODI), Roland-Morris (RM) y Escala Analógica Visual (VAS). Métodos. Fueron evaluados los cambios en los dados de los cuestionarios (ODI, RM y VAS) entre el preoperatorio, 1 sin, 1 mes, 2 meses, 6 meses, 1 año y 2 años después del procedimiento quirúrgico. Resultados. Fueron estudiados 38 pacientes con edades de más de 60 años, con acompañamiento de, por lo menos, dos años. Hubo una mejora en el ODI de 55,94\%, 49,18\% en el RM y 39,48\% en el VAS. Conclusión. Los resultados muestran una mejora en los índices analizados, presentando índices semejantes a los de estudios anteriores, a pesar de la discrepancia entre los diversos estudios y la falta de datos referentes a nuestra población.

Descriptores: Artrodesis; Vértebras lumbares; Fusión vertebral; Calidad de vida.

\section{INTRODUÇÃO}

O avanço da medicina e o melhor conhecimento da prevenção de doenças em relação à alimentação, exercícios e acompanhamento médico adequado têm aumento à expectativa de vida da população. A incidência da população americana acima de 75 anos em 2006 foi de $6 \%$ da população e a projeção para 2050 é o dobro desse valor ${ }^{1}$. Esse grupo de indivíduos cada vez mais é ativo realizando inclusive atividades esportivas não somente de lazer.

Alguns desses indivíduos desenvolverão estenose de canal lombar, espondilostese degenerativa sintomática que falharão com o tratamento conservador. Com a evolução de técnicas cirúrgicas e instrumentais houve um aumento no número de procedimentos cirúrgicos ${ }^{2,3}$, sendo que nos Estados Unidos houve um aumento de
100\% nos últimos 10 anos $^{4}$. A descompressão cirúrgica associada à artrodese posterolateral tem sido o tratamento de escolha para essas afecções.

A satisfação do paciente é uma importante ferramenta na avaliação de resultados ${ }^{5}$. Estudos recentes estão levando em consideração medidas subjetivas de sintomas e questionários de qualidade de vida do paciente $e^{6-8}$. Poucos artigos têm sido publicados avaliando o impacto desses procedimentos cirúrgicos na população geriátrica.

O objetivo deste estudo é avaliar a percepção de melhora na qualidade de vida de pacientes submetidos a uma cirurgia de descompressão e artrodese lombar através dos questionários Owestry Disability Index (ODI), Roland-Morris e a Escala Analógica Visual para dor (VAS) neste grupo de pacientes.

1. Médico Ortopedista e Estagiário em Cirurgia de Coluna da Faculdade de Medicina do ABC - Santo André, SP, Brasil.

2. Doutor em Ciências Médicas e Professor Adjunto da Cadeira de Ortopedia eTraumatologia da Faculdade de Medicina do ABC - Santo André, SP, Brasil.

Trabalho realizado no Hospital Mário Covas, Santo André - SP (Faculdade de Medicina do ABC).

Correspondência: Av. Príncipe de Gales, 821 - Príncipe de Gales - 09060-650 - Santo André, SP, Brasil. spine.yoshino@gmail.com 


\section{MATERIAIS E MÉTODOS}

Após receber a aprovação no comitê de ética da instituição, foram estudados 41 pacientes que apos um acompanhamento de, pelo menos seis meses de tratamento conservador de estenose de canal lombar no ambulatório de coluna no Hospital Mario Covas e após a falha no tratamento instituído foram submetidos a cirurgia de artrodese lombar associada à descompressão no período de maio de 2003 a dezembro de 2008. Com base na coleta dos dados foi elaborado um estudo de coorte retrospectivo comparando os resultados através dos questionários VAS,ODI e Roland-Morris. As cirurgias foram realizadas por dois cirurgiões experientes. Todas as cirurgias foram realizadas no Hospital Mario Covas, sediado em Santo André - SP. Dados como idade, sexo e data da cirurgia foram retirados do prontuário dos pacientes. Após a indicação cirúrgica do paciente, o mesmo era dirigido à outra sala onde era submetido aos questionários de Escala Analógica Visual de Dor (VAS), Oswestry Disability Index (ODI) e Roland-Moris realizados por uma psicóloga. Realizado o procedimento e após a alta hospitalar todos pacientes eram encaminhados ao ambulatório do serviço e após a consulta ambulatorial de rotina (sétimo pós-operatório), se dirigiam a outra sala, a mesma psicóloga aplicava os mesmos questionários (VAS, ODI e Roland-Moris). Esta rotina foi repetida nas consultas após um mês, seis meses, um ano e dois anos. Os resultados eram registrados no prontuário. Os resultados eram registrados e arquivados no prontuário sendo este guardados no serviço de arquivo médico e estatístico (SAME) do hospital e através da análise dos prontuários foi elaborado o estudo feita por um médico experiente. Apenas pacientes portadores de doenças degenerativas acima de 60 anos foram incluídos neste estudo. Portadores de tumores, outras patologias degenerativas associadas (ex.: espondilolistese), infecções, fraturas, revisões, com o questionário incompleto e menos de 60 anos foram excluídos do estudo.

O ODI (traduzido e adaptado para o Português) e um questionário baseado nas restrições sociais e físicas que a dor nas costas proporciona. Contem 10 questões que cobrem diferentes atividades diárias. O resultado varia de 0\% a 100\%, sendo 0\% indicando sem restrições e $100 \%$ a limitação máxima ${ }^{9}$.

O VAS e um questionário onde o paciente avalia e classifica sua dor dando uma nota que varia de zero a dez, sendo uma ferramenta de avaliação subjetiva nos sintomas de dor crônica ${ }^{10}$.

O questionário Roland-Morris foi criado em 1983 para avaliar os impactos que a dor lombar interferia nas atividades cotidianas do paciente. Composto de 24 questões onde cada questão é atribuída um ponto e quanto maior a somatória, maior será a incapacidade do indivíduo ${ }^{11}$.

\section{ANALISES ESTATÍSTICA}

Usamos o programa SPSS (Statistical Package for Social Sciences $\left(B M^{\circledR}\right)$, em sua versão 19.0, para a obtenção dos resultados. A aplicação do Teste de Friedman foi o método usado para calcular as variações. Como foram encontradas diferenças estatisticamente significantes, para as três variáveis de interesse, vamos aplicar o Teste dos Postos Sinalizados de Wilcoxon, ajustado pela Correção de Bonferroni, com o intuito de tentarmos identificar quais momentos de observação diferem-se entre si, quando comparados par a par (Tabela 1). Adotamos o nível de significância de 5\% (0,050), para a aplicação dos testes estatísticos.

\section{RESULTADOS}

Um total de 38 pacientes participaram do estudo, sendo acompanhados e avaliados de setembro de 2002 até novembro de 2010. A população era constituída de 17 mulheres (44,73\%) e 21 homens $(55,27 \%)$ acima de 60 anos (idade média de 68,3 anos). Todos os pacientes foram submetidos a uma descompressão associada artrodese instrumentada.

Houve uma melhora estatisticamente significante em todos os parâmetros estudados. A variação média do ODI melhorou de 61,04 no pré-operatório para 34,15 após dois anos (Figura 1). No questionário Roland-Morris apresentou uma redução de 17,14 para 8,43 (Figura 2). No VAS mostra também uma diminuição, com variação de 8,84 para 3,49 (Figura 3). Em todos os questionários houve uma latência entre os períodos entre um e dois anos.
Tabela 1. Significância dos três critérios avaliados.

\begin{tabular}{c|c|c|c}
\hline \multirow{2}{*}{ Par de Variáveis } & \multicolumn{3}{|c}{ Significância $(\mathbf{p})$} \\
\cline { 2 - 4 } & Oswestry & RM & VAS \\
\hline $1 \mathrm{~m}$ - pré & 0,829 & 0,025 & $<0,001$ \\
\hline $6 \mathrm{~m}$ - pré & 0,015 & $<0,001$ & $<0,001$ \\
\hline $1 \mathrm{a}$ - pré & $<0,001$ & $<0,001$ & $<0,001$ \\
\hline $2 a-$ pré & $<0,001$ & $<0,001$ & $<0,001$ \\
\hline $6 \mathrm{~m}-1 \mathrm{~m}$ & 0,005 & 0,001 & $<, 002$ \\
\hline $1 \mathrm{a}-1 \mathrm{~m}$ & $<0,001$ & $<0,001$ & 0,001 \\
\hline $2 a-1 m$ & $<0,001$ & $<0,001$ & 0,002 \\
\hline $1 a-6 m$ & 0,008 & 0,011 & 0,141 \\
\hline $2 a-6 m$ & 0,008 & 0,015 & 0,408
\end{tabular}

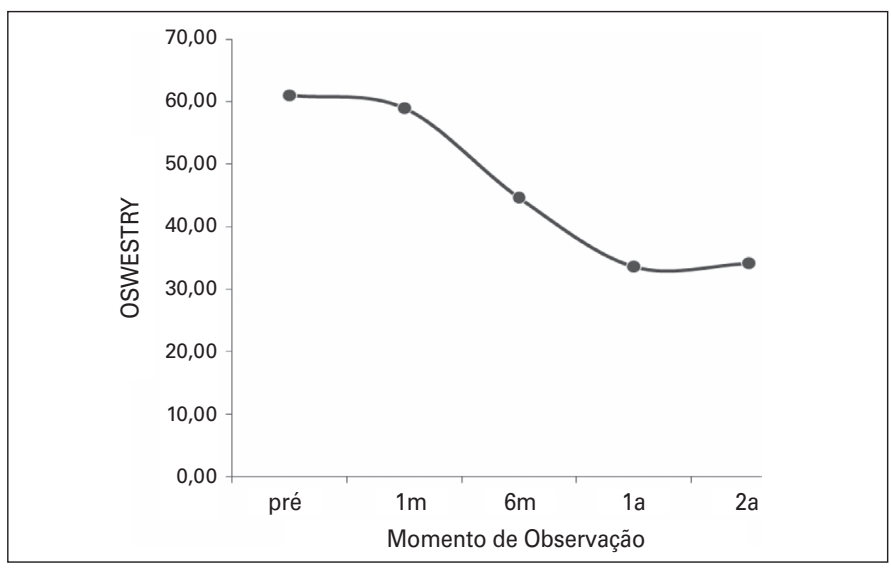

Figura 1. Evolução dos questionários ODI.

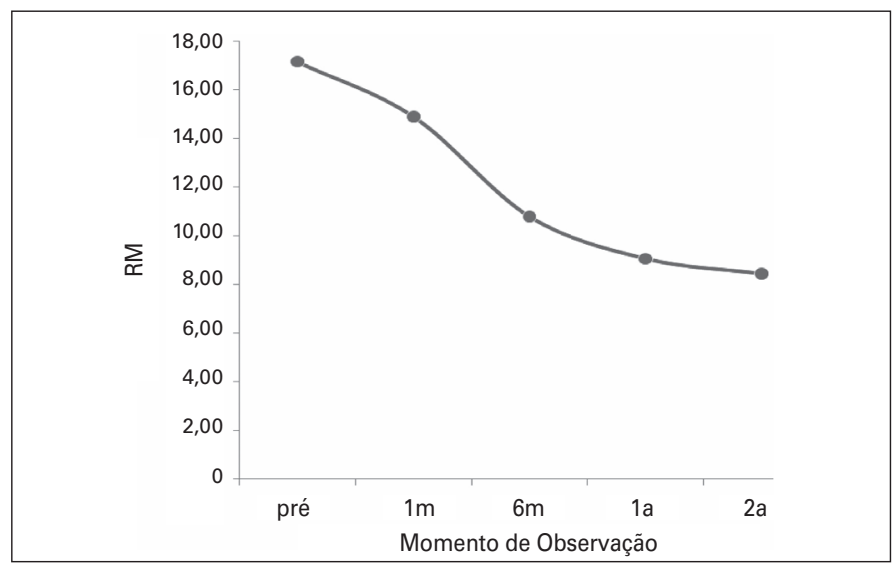

Figura 2. Evolução dos questionários RM.

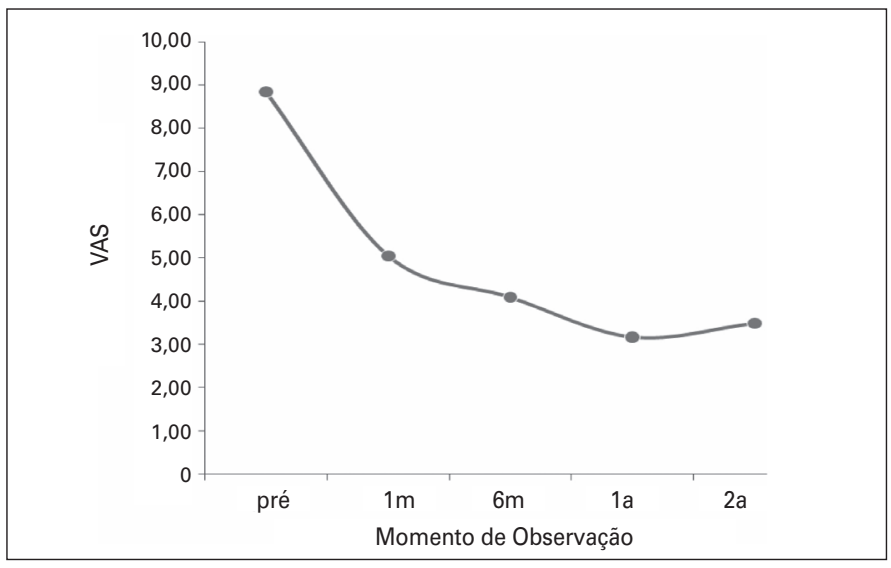

Figura 3. Evolução dos questionários VAS. 


\section{DISCUSSÃO}

A satisfação é de suma importância na análise dos resultados ${ }^{5}$. Historicamente, o sucesso de uma artrodese era medido apenas pelo grau de artrodese. Estudos recentes estão levando em consideração medidas subjetivos de sintomas e questionários de qualidade de vida do paciente ${ }^{6-8}$. Instrumentos de avaliação de parâmetros subjetivos são necessários para avaliar o estado de saúde do paciente e consequentemete os resultados das cirurgias. Fenômenos objetivos tais como amplitude de movimento, deformidades e sensibilidade e subjetivos como angustia e dor são agrupados em grupos e subgrupos e permitem a quantificacão de determinados componentes do estado de saúde ${ }^{12}$. Questionários como o VAS, RM e ODI são rotineiramente usados na avaliação e acompanhamento de procedimentos cirúrgicos na coluna lombar ${ }^{13}$.

Apesar dos estudos anteriores mostrarem que os resultados do tratamento cirúrgico quando bem indicado são superiores ao tratamento conservador ${ }^{14-17}$, a avaliação dos resultados na cirurgia da coluna continua sendo um desafio. Discrepâncias entre os resultados de diversos trabalhos na literatura comprovam. Estudos como de Ragab et al. ${ }^{18}$ mostram índices de 92\% de melhora pós-operatória. Chen et al. ${ }^{19}$ reportam resultados bom-excelentes de $77 \%$ dos pacientes estudados após 5 anos de acompanhamento. Albert et al. ${ }^{20}$ apresentam índices de melhora de 65\% dos pacientes com taxas de pseudoartrose de $10 \%$. Nosso estudo encontrou resultados semelhantes e compatíveis com a literatura com significativa melhora em todos os índices avaliados, evoluindo para um cotidiano mais funcional, uma vez que a capacidade funcional é determinada pelo grau de função e habilidade física, assim sendo, ocorreu importante diminuição das limitações. Os pacientes estudados obtiveram diminuição da pontuação de 17,14 $( \pm 4,24)$ no RM e 61,04( $\pm 18,39)$ no ODI para 8,43( $\pm 4,3)$ e 34,15 ( $\pm 17,82)$, respectivamente após 2 anos da cirurgia. A escala analógica de dor revelou diminuição na intensidade da dor de 8,84 \pm 1,74 para $3,49 \pm 2,71$, o que também pode ser indicativo de aumento da funcionalidade, pois um quadro álgico é altamente incapacitante.

A maioria dos estudos segue um padrão de, pelo menos, dois anos de acompanhamento para avaliação de resultados, o que valida ainda mais este estudo. Os parâmetros vão melhorando gradativamente através de um ano de acompanhamento e se estabilizam, mantendo índices constantes após dois anos, mostrando tanto a eficácia do procedimento quanto a manutenção dos bons resultados em longo prazo.

\section{REFERÊNCIAS}

1. National Center for Health Statistics Health, United States, 2008 with Chartbook Hyattsville, MD. 2009. Disponível em: http://www.cdc.gov/nchs/hus.htm. Acesso em 19 de outubro,2009

2. Bono CM, Lee CK. Critical analysis of trends in fusion for degenerative disc disease over the past 20 years: influence of technique on fusion rate and clinical outcome. Spine (Phila Pa 1976). 2004;29(4):455-63

3. Mardjetko SM, Connolly PJ, Shott S. Degenerative lumbar spondylolisthesis. A meta-analysis of literature 1970-1993. Spine (Phila Pa 1976). 1994;19(20 Suppl):2256S-2265S

4. Taylor VM, Deyo RA, Cherkin DC, Kreuter W. Low back pain hospitalization. Recent United States trends and regional variations. Spine (Phila Pa 1976). 1994;19(11):1207-12.

5. Sinikallio S, Aalto T, Airaksinen O, Herno A, Kröger H, Savolainen $\mathrm{S}$, et al. Lumbar spinal stenosis patients are satisfied with short-term results of surgery - younger age, symptom severity, disability and depression decrease satisfaction. Disabil Rehabil. 2007:29(7):537-44

6. Glassman S, Gornet MF, Branch C, Polly D Jr, Peloza J, Schwender JD, et al. MOS short form 36 and Oswestry Disability Index outcomes in lumbar fusion: a multicenter experience. Spine J. 2006:6(1):21-6

7. Weiner BK. Re:Walsh TL, Hanscom B, Lurie JD, et al. Is a condition-specific instrument for patients with low back pain/leg symptoms really necessary? The responsiveness of the Oswestry Disability Index, MODEMS, and the SF-36. Spine. 2003;28:607-15.

8. Fairbank JC, Pynsent PB. The Oswestry Disability Index. Spine (Phila Pa 1976). 2000;25(22):2940-52.

9. Fairbank JC, Couper J, Davies JB, O'Brien JP. The Oswestry low back pain disability questionnaire. Physiotherapy. 1980;66(8):271-3.

10. Price DD, McGrath PA, Rafii A, Buckingham B. The validation of visual analogue scales as ratio scale measures for chronic and experimental pain. Pain. 1983;17(1):45-56.

11. Nusbaum L. Tradução, adaptação e validação do Questionário Roland-Morris: Brazil Roland-Morris (Brazil RM). [tese]. São Paulo: Universidade Federal de São Paulo 1996

12. Boden SD. Outcome assessment after spinal fusion: why and how? Orthop Clin North Am. 1998;29(4):717-28.
Os questionários de qualidade de vida que são instrumentos de avaliação são complementares e importantes para verificar a eficácia do tratamento cirúrgico e a capacidade funcional dos participantes, assim como direcionar tratamentos mais específicos para a aquisição de maiores ganhos. Uma vez que todos os participantes obtiveram algum ganho em funcionalidade e qualidade de vida, foram concebidos a fim de promover uma reprodutibilidade, mas eventualmente diferenças interobservadores podem ocorrer. Este fato foi minimizado no estudo em questão, onde todos os questionários foram feitos por um mesmo psicólogo, diminuindo assim possíveis vieses neste aspecto.

Encontramos dificuldades de estabelecer a idade mínima como critério de inclusão, pois em diversos estudos a idade foi estabelecida por critérios arbitrários. Alguns autores estabeleceram como sessenta e cinco anos ${ }^{21,22}$, enquanto outros escolheram sessenta $\operatorname{anos}^{21,23,24}$. Com os avanços da medicina e o aumento da longevidade estabelecer uma idade de corte é uma difícil tarefa, mas uma padronização seria imprescindível para uma melhor comparação de resultados entre os diversos estudos.

Infelizmente existem poucos estudos ou dados estatísticos em âmbito nacional sobre a incidência de dores lombares na população brasileira, bem como suas repercussões: índice de afastamento do trabalho; acesso ao atendimento da saúde; realização de exames complementares e procedimentos; número de recorrências. Dados como esses relacionados seriam de grande valia na compreensão do impacto econômico e social que as doenças degenerativas exercem sobre a sociedade brasileira.

Devemos decifrar e compreender as doenças degenerativas devido ao aumento da expectativa de vida da população mundial, essas patologias estão ganhando cada vez mais importância, pois o adequado manejo das doenças relacionadas ao envelhecimento devem ser estudadas e compreendidas, a fim de garantir uma melhor qualidade de vida para esses pacientes. Este estudo visa corroborar os fortes indícios da eficácia no aumento sobre a percepção do paciente em sua qualidade de vida através da artrodese lombar nas doenças degenerativas da coluna vertebral.

\section{CONCLUSÃO}

Segundo este estudo, a artrodese posterolateral da coluna se mostra eficaz na melhora dos parâmetros subjetivos. Houve uma melhora gradativa e estatisticamente significante em todos os questionários estudados em até 1 ano, com manutenção dos índices em 2 anos de acompanhamento das patologias em questão.

13. Carreon LY, Glassman SD, Djurasovic M, Dimar JR, Johnson JR, Puno RM, et al. Are preoperative health-related quality of life scores predictive of clinical outcomes after lumbar fusion? Spine (Phila Pa 1976). 2009;34(7):725-30

14. Atlas SJ, Keller RB, Wu YA, Deyo RA, Singer DE. Long-term outcomes of surgical and nonsurgical management of lumbar spinal stenosis: 8 to 10 year results from the maine lumbar spine study. Spine (Phila Pa 1976). 2005;30(8):936-43

15. Sengupta DK, Herkowitz HN. Lumbar spinal stenosis. Treatment strategies and indications for surgery. Orthop Clin North Am. 2003;34(2):281-95.

16. Amundsen T, Weber H, Nordal HJ, Magnaes B, Abdelnoor M, Lilleâs F. Lumbar spinal stenosis: conservative or surgical management?: A prospective 10-year study. Spine (Phila Pa 1976). 2000;25(11):1424-35.

17. Filho EVS, Ueno FH, Cabral LTB, Yonezaki AM, Nicolau RJ, Rodrigues LMR. Estudo prospectivo de avaliação de dor e incapacidade de pacientes operados de estenose de canal lombar com seguimento mínimo de dois anos . Coluna/Columna. 2009:8(4):390-4

18. Ragab AA, Fye MA, Bohlman HH. Surgery of the lumbar spine for spinal stenosis in 118 patients 70 years of age or older. Spine (Phila Pa 1976). 2003;28(4):348-53.

19. Chen WJ, Lai PL, Niu CC, Chen LH, Fu TS, Wong CB. Surgical treatment of adjacent instability after lumbar spine fusion. Spine (Phila Pa 1976). 2001;26(22):E519-24.

20. Albert TJ, Pinto M, Denis F. Management of symptomatic lumbar pseudarthrosis with anteroposterior fusion. A functional and radiographic outcome study. Spine (Phila Pa 1976). 2000;25(1):123-9

21. Glassman SD, Carreon LY, Dimar JR, Campbell MJ, Puno RM, Johnson JR. Clinical outcomes in older patients after posterolateral lumbar fusion. Spine J. 2007:7(5):547-51.

22. Glassman SD, Polly DW, Bono CM, Burkus K, Dimar JR. Outcome of lumbar arthrodesis in patients sixty-five years of age or older. J Bone Joint Surg Am. 2009;91(4):783-90.

23. Okuda S, Oda T, Miyauchi A, Haku T, Yamamoto T, Iwasaki M. Surgical outcomes of posterior lumbar interbody fusion in elderly patients. J Bone Joint Surg Am. 2006;88(12):2714-20.

24. TokuhashiY, Ajiro Y, Umezawa N. Outcomes of posterior fusion using pedicle screw fixation in patients >or=70 years with lumbar spinal canal stenosis. Orthopedics. 2008;31(11):1096 